\title{
The unveiled reality of human papillomavirus as risk factor for oral cavity squamous cell carcinoma
}

\author{
Irene H. Nauta ${ }^{1}$ (1) | Daniëlle A. M. Heideman ${ }^{2}$ | Arjen Brink ${ }^{1}$ | \\ Berdine van der Steen $^{3}$ | Elisabeth Bloemena ${ }^{2,4}$ | Senada Koljenović ${ }^{5}$ | \\ Robert J. Baatenburg de Jong ${ }^{3}$ | C. René Leemans ${ }^{1}$ | Ruud H. Brakenhoff ${ }^{1}$
}

${ }^{1}$ Amsterdam UMC, Vrije Universiteit Amsterdam, Otolaryngology/Head and Neck Surgery, Cancer Center Amsterdam, Amsterdam, The Netherlands

${ }^{2}$ Amsterdam UMC, Vrije Universiteit Amsterdam, Pathology, Cancer Center Amsterdam, Amsterdam, The Netherlands

${ }^{3}$ Department of Otorhinolaryngology/Head and Neck Surgery, Erasmus Medical Center, Erasmus MC Cancer Institute, Rotterdam, The Netherlands

${ }^{4}$ Department of Maxillofacial Surgery/Oral Pathology, Academic Medical Centre for Dentistry, Amsterdam, The Netherlands

${ }^{5}$ Department of Pathology, Erasmus Medical Center, Erasmus MC Cancer Institute,

Rotterdam, The Netherlands

\section{Correspondence}

Ruud H. Brakenhoff, Department of Otolaryngology/Head and Neck Surgery, Tumor Biology Section, Amsterdam UMC, location VUmc, De Boelelaan 1117, PO Box 7057, 1007 MB Amsterdam, The Netherlands. Email: rh.brakenhoff@amsterdamumc.nl

Funding information Horizon 2020 Framework Programme, Grant/ Award Number: Grant Agreement 689715 BD2Decide

\begin{abstract}
The prognostic impact of human papillomavirus (HPV) in oropharyngeal cancer is generally acknowledged, and HPV-status is assessed routinely in clinical practice. Paradoxically, while the oral cavity seems the predilection site for productive HPVinfections, figures on HPV-attribution in oral cavity squamous cell carcinoma (OCSCC) differ widely, and prognostic impact is uncertain. Major obstacles are the lack of reproducible assays to detect HPV in nonoropharyngeal cancers, the relatively small cohorts studied and consequently the shortfall of convincing data. In our study, we used a validated, nucleic acid-based workflow to assess HPV-prevalence in a consecutive cohort of 1016 OCSCCs, and investigated its prognostic impact. In parallel, we analyzed $\mathrm{p} 16$-immunohistochemistry $(\mathrm{p} 16-\mathrm{IHC})$ as surrogate marker for transforming HPV-infection and independent prognosticator. All OCSCC-patients diagnosed between 2008 and 2014 at two Dutch university medical centers were included $(N=1069)$. Formalin-fixed, paraffin-embedded (FFPE)-samples of 1016 OCSCCs could be retrieved. Punch biopsies were taken from the tumor area in the FFPEblocks and tested for HPV. P16-IHC was performed on 580 OCSCCs, including all HPV-positive tumors. From 940 samples (92.5\%), nucleic acids were of sufficient quality for HPV-testing. In total, 21 (2.2\%) OCSCCs were HPV DNA-positive. All HPV DNA-positive tumors were E6 mRNA-positive and considered as true HPV-positive. There was no difference in survival between HPV-positive and HPV-negative OCSCCs. In total, 46 of 580 (7.9\%) OCSCCs were p16-immunopositive, including all HPV-positive tumors. Survival was comparable in p16-positive and p16-negative OCSCCs. To conclude, HPV-prevalence is very low in OCSCC and neither HPVstatus nor p16-status affects outcome. Based on these data, determining HPV-status in OCSCC seems irrelevant for clinical management.
\end{abstract}


KEYWORDS

human papillomavirus, oral cavity squamous cell carcinoma, p16-immunohistochemistry, prevalence, prognosis

\section{1 | INTRODUCTION}

Infection with high-risk human papillomavirus (HPV) is an important risk factor for head and neck squamous cell carcinoma (HNSCC). Although HPV-attribution varies between geographical regions, the oropharynx is consistently identified as the head and neck site with the highest frequency of HPV-related squamous cell carcinomas (SCCs), particularly in the Western world. ${ }^{1-3}$ Within the oropharynx, HPV-related tumors are most abundant in the lingual tonsils and base of tongue, ${ }^{4}$ possibly due to the reticular crypt epithelial cells that may have a high oncogenic transforming potential. ${ }^{5}$ Compared to HPVnegative tumors, HPV-related oropharyngeal squamous cell carcinomas (OPSCCs) differ in both patient and tumor characteristics, ${ }^{6,7}$ and have a significantly more favorable prognosis when treated with standard of care chemoradiotherapy.,9 HPV-related OPSCCs are therefore considered a distinct disease entity with a separate UICC TNM classification since the eighth edition. ${ }^{10}$

Although the prevalence and prognostic impact of HPV in OPSCC is undisputable, its role in nonoropharyngeal HNSCCs remains unclear. In the hypopharynx and larynx, the general consensus is that the proportion of HPV-positive tumors seems relatively low (1.6\%-6.4\% and 1.6\%-7.9\%, respectively). ${ }^{1,11-13}$ The major issue lies within oral cavity squamous cell carcinomas (OCSCCs). Significant differences in HPV-attribution for OCSCCs have been reported, ranging from $2.0 \%$ to frequencies as high as $61.0 \% .^{1,2,12,14-22}$ Apart from geographical variation and a relative increase in HPV-prevalence over time, several other factors might specifically contribute to this discordance. First of all, tumor site misclassification between the oral cavity and oropharynx may play a role in literature. Carcinomas arising from the oropharynx are occasionally referred to as oral cancers, and the base of tongue is sometimes considered part of the oral cavity, ${ }^{23}$ resulting in higher HPV-attributable fractions than truly is the case. As the anatomical sites have been well-defined, ${ }^{23}$ this should be easy to avoid. Nevertheless, larger tumors that overlap between the oropharynx and oral cavity may have found its origin in either of these sites, and thereby may confound figures.

Most importantly, however, is that there is no universal HPV-testing method available for nonoropharyngeal HNSCCs. The various HPV DNA polymerase chain reaction (PCR)-assays that are typically used are generally developed for cervical cancer screening and prevalence studies of cervical HPV-infection. These very sensitive assays may detect not only transforming HPV-infections, but also HPV DNA from productive infections. These assays are also more susceptible to contamination, leading to false-positive results. ${ }^{24,25}$ Detection of HPV E6/E7 transcripts is therefore considered the gold standard for transforming infections in all HNSCC sites, ${ }^{26}$ but this test is generally not feasible in daily practice as regular RNA-based assays usually demand fresh-frozen tumor material, which is not routinely collected. Therefore, alternative methods have been proposed. For OPSCC, a test

\section{What's new}

While human papillomavirus (HPV)-infection is prognostic for oropharyngeal squamous cell carcinoma (OCSCC), its role and prognostic impact in OCSCC remain uncertain. In the present study, nucleic acid-based HPV-testing of a cohort of 1016 tumor samples collected from OCSCC patients diagnosed between 2008 and 2014 reveals very low HPV-prevalence in OCSCC. In addition, no difference was found in survival between HPV-positive and HPV-negative tumors. The findings indicate that HPV is uncommon in OCSCC and, when present, has no impact on survival, providing new and important insight into the ongoing debate regarding the role of HPV in OCSCC.

algorithm for formalin-fixed, paraffin-embedded (FFPE) specimen was developed by Smeets et al, consisting of p16-immunohistochemistry (p16-IHC), followed by high-risk HPV DNA-detection with GP5+/6 +-PCR on p16-immunopositive cases. ${ }^{26}$ The p16 protein, formally $\mathrm{p} 16^{\text {Ink4A }}$, is encoded by the CDKN2A gene, a frequently inactivated tumor suppressor gene in HPV-negative HNSCC. It typically shows increased expression in HPV-positive tumors as a functional consequence of HPV E7 oncogene signaling, making it an excellent surrogate marker for a transforming HPV-infection, at least in OPSCC. Validation of the HPV test algorithm on an external cohort resulted in an accuracy of $98 \%$ when compared to detection of HPV E6 oncogene expression in frozen tumor specimens as gold standard for a transforming HPV-infection. ${ }^{27}$ Unfortunately, this test algorithm does not perform well on OCSCCs with a positive-predictive value of only 41.3\%. ${ }^{16}$ In 2019, Mes et al developed a rapid and contamination-free HPV-test method suited for FFPE-material from both OPSCC and non-OPSCC, called the HPV-rTcore workflow. ${ }^{28}$ This method circumvents sectioning of FFPE-specimens on the microtome by taking hemotoxylin and eosin (H\&E)-guided core punch biopsies from the tumor area in the FFPE-blocks. From these biopsies, total nucleic acids are isolated, which are subsequently subjected to testing for the presence of HPV DNA by targeting the E7 region of 15 high-risk HPV-types, followed by testing for the presence of type-specific HPV E6 mRNA in case of an HPV DNA-positive test result. Validation of the HPV-rTcore workflow on independent cohorts of OPSCCs and OCSCCs resulted in an accuracy of near $100 \%$ when compared to the earlier mentioned gold standard of HPV E6 transcript detection on RNA isolated from fresh-frozen tumor specimens. ${ }^{28}$

The aim of our study was to determine the HPV-attributable fraction in a large consecutive cohort of OCSCCs by using the validated 
HPV-rTcore workflow, and to investigate the prognostic impact of HPV-positivity in OCSCC. Although p16-IHC is considered an inadequate surrogate marker for a transforming HPV-infection in OCSCC, ${ }^{16}$ a survival benefit of p16-positivity has been described for nonOPSCC irrespective of HPV-status. ${ }^{12,29}$ The secondary aim of our study was therefore to assess the performance of $\mathrm{p} 16-\mathrm{IHC}$ as surrogate marker for a transforming HPV-infection, as well as its role as independent prognosticator in OCSCC.

\section{2 | MATERIALS AND METHODS}

\subsection{Study design}

To determine the required sample size for our study, a sample size calculation was performed first. It was assumed that the true HPV-attributable fraction in OCSCC was between $5 \%$ and $10 \%$, and therefore we assumed an HPV-proportion of $7.5 \%$. With an $\alpha$ of .05, a $\beta$ of .2, an HPV-proportion of 7.5\%, a hazard of 2.0, 15\% baseline events per year, a censoring rate of $10 \%$ per year and an average follow-up period of 2 years, we had to include 942 patients in the analysis. Therefore, it was decided to carry out this retrospective study on a consecutive cohort of TNM-8 stage I-IVC OCSCC patients diagnosed between 2008 and 2014 in Amsterdam UMC, location VUmc and Erasmus MC Rotterdam ( $N=1069$ ). Patients were identified through the Dutch Cancer Registries, and clinical data collected from the patient files. Squamous cell carcinomas of the external lip (ICD-10 codes C00.0-C00.2 and C00.6-C00.9) were excluded, while all other OCSCC subsites were included (ICD-10 codes C00.3-C00.5, C02.0-C02.3, C02.8-C05.0, C06.0-C06.9). Of note, the oral cavity is located directly adjacent to the oropharynx, so tumors arising in the oral cavity might expand to the oropharynx and vice versa, making it challenging to establish the site of origin when tumors cross the anatomical borders. Considering that the majority of HPV-positive head and neck cancers are detected in the oropharynx, site misclassification might result in inaccurate HPVattributable fractions for OCSCCs. We therefore excluded all overlapping lesions of the oral cavity and oropharynx, either on basis of the corresponding ICD-10 codes (C05.8 "overlapping lesion of palate" and C14.8 "overlapping lesion of lip, oral cavity and pharynx," $N=7$ ) or on basis of reports from physical examination including panendoscopy and/or imaging that the tumor crossed the anatomical border ( $N=15)$. FFPE-tissue from 1016 of the 1069 tumors (95\%) could be retrieved from the pathology archives. HPV-attributable fraction was assessed based on all 1016 OCSCC patients, while the effect of HPV-status on OCSCC prognosis was analyzed only on OCSCC patients treated with curative intent $(\mathrm{N}=955)$.

Demographic and clinical characteristics, as well as survival outcomes were obtained from the patient files. A pack year was defined as smoking 20 cigarettes a day during 1 year. A unit year was defined as consuming one alcoholic unit a day during 1 year. Histopathological features of all tumors were scored by dedicated head and neck pathologists (E. Bloemena, S. Koljenović) according to the WHO Classification of Head and Neck Tumours. In a recently published article on HPV-positive tumors of the hypopharynx, larynx and oral cavity, three clinicopathological variants (keratinizing, nonkeratinizing and warty $)^{30}$ were described, and these classifications were scored as well.

\section{2 | HPV-testing}

HPV-status was determined on the FFPE-samples taken for routine diagnostic workup. HPV-status was assessed by the HPV-rTcore workflow as described by Mes et al. ${ }^{28}$ In short, a core biopsy of $1 \mathrm{~mm}$ diameter was taken from the tumor area of each FFPE-specimen by guidance of the corresponding H\&E-slide using disposable biopsy punches with a plunger system (Kai Europe $\mathrm{GmbH}$, Solingen, Germany). DNA and RNA were simultaneously isolated from the core biopsies using the NucliSENS easyMag (bioMérieux, Marcy-l'Étoile, France). Each isolate was first tested for the presence of HPV DNA either using a quantitative real-time PCR assay targeting the E7 region of 15 high-risk HPV-genotypes: HPV type 16, 18, 31, 33, 35, 39, 45, 51, 52, 56, 58, 59, 66, 67 and 68 (HPV-Risk Assay [Self-screen B.V., Amsterdam, the Netherlands] on the ViiA 7 real-time PCR system [Applied Biosystems, Foster City, CA]; or using the equivalent QIAscreen HPV PCR Test [QIAGEN, Hilden, Germany] on the Rotor-Gene $Q$ real-time PCR cycler [QIAGEN]). ${ }^{31}$ Four fluorescent channels were used: one for HPV16, one for HPV18, one for the other 13 (probable) high-risk HPV-types and one for the human $\beta$-globin gene. The latter served as internal control to confirm the presence of DNA with sufficient quality for reliable HPV DNA test results. For the ViiA 7, threshold cycle threshold values (Ctvalues) were $\leq 36$ for all four channels. ${ }^{28}$ For the Rotor-Gene $\mathrm{Q}$, threshold Ct-values were $\leq 36$ for HPV16 and HPV18, $\leq 33.5$ for the other 13 (probable) high-risk HPV-types and $\leq 30$ for $\beta$-globin. The two assay versions were calibrated against each other and are used in diagnostics.

All HPV DNA-positive OCSCC were subsequently tested for HPV type-specific E6 mRNA with assays specifically designed for FFPE (ie, degraded RNA; Supplementary Table 1). Genotype information was derived from the real-time PCR assay for HPV16 and HPV18, and from additional testing with GP5+6+-PCR luminex for samples that were reported positive for the other 13 (probable) high-risk HPVtypes. $^{32}$ In addition, 50 HPV DNA-negative OCSCCs were tested for HPV16 E6 mRNA to exclude false-negative HPV DNA test results. An OCSCC was considered truly HPV-positive if both the HPV DNA and HPV E6 mRNA were demonstrated (Figure 1).

\section{3 | p16-immunohistochemistry}

To determine the reliability of $\mathrm{p} 16-\mathrm{IHC}$ as surrogate marker for a transforming HPV-infection in OCSCC, as well as its role as independent prognosticator, p16-IHC was performed on all HPV-positive OCSCCs and 559 randomly selected HPV-negative OCSCCs. P16-status was assessed by one of two experienced head and neck pathologists independently and without knowledge of HPV-status. Equivocal staining results were evaluated by the second head and neck pathologist. Similar as in OPSCC, 9 a cutoff value of $\geq 70 \%$ strong and diffuse nuclear and cytoplasmic immunostaining of p16 was used. All tumors that did not meet this criterion were considered p16-negative. Of note, it is not 


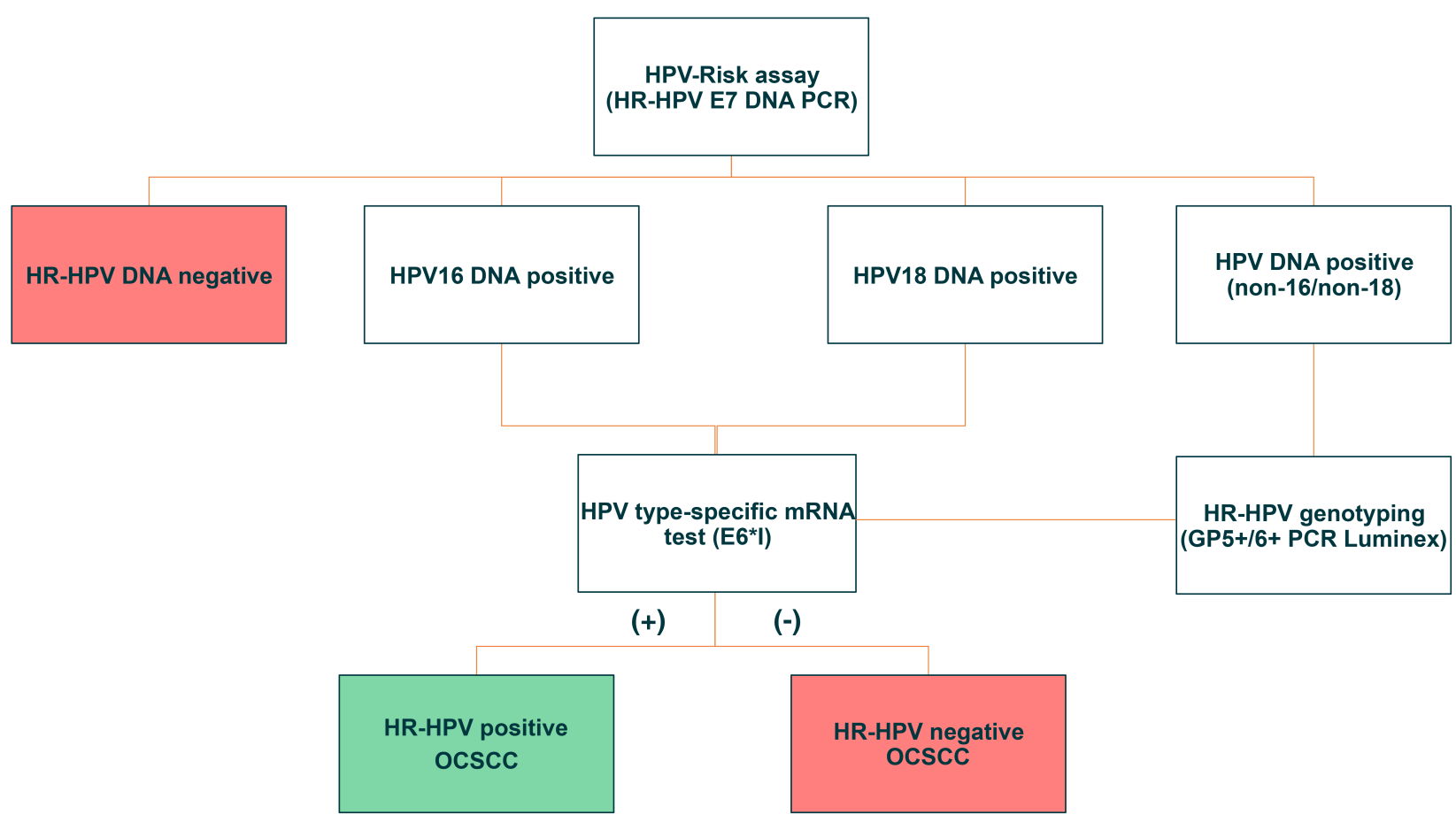

FIGURE 1 Human papillomavirus (HPV) test algorithm for oral cavity squamous cell carcinoma (OCSCC) [Color figure can be viewed at wileyonlinelibrary.com]

known whether this cutoff value, which was specifically defined for OPSCC, is valid for OCSCC as well. We therefore did not only score each tumor as being either p16-negative or p16-positive, but we paid extra attention to potential variations in staining pattern.

\subsection{Clinical endpoints}

Clinical endpoints for our study were based on the "Clinical Trial Endpoints for the Approval of Cancer Drugs and Biologics" by the Food and Drug Administration (https://www.fda.gov/media/71195/ download) and included 5-year overall survival (OS) and disease-free survival (DFS). OS was defined as the time from histologically confirmed OCSCC diagnosis until death from any cause. DFS was defined as the time from histologically confirmed OCSCC diagnosis until disease recurrence or death from any cause. Disease recurrence included local recurrence (within $2 \mathrm{~cm}$ and within 3 years of diagnosis of the index tumor), regional recurrence (lymph node metastases in previously treated levels of the neck) and distant metastases. Patients with residual disease, delayed lymph node metastases or second primary tumors were censored at the respective dates.

\subsection{Statistical analysis}

Differences in demographic and clinical characteristics between de HPV-positive and HPV-negative OCSCCs were determined using the Pearson chi-square test or Fisher's exact test for categorical variables, and the Student's $t$ test for continuous variables. OS and DFS curves were visualized using Kaplan-Meier plots, and strata compared with the long-rank test. $P$-values $<.05$ were considered statistically significant. Analyses were performed with IBM SPSS Statistics 26.

\section{3 | RESULTS}

\section{1 | Prevalence of HPV in OCSCC}

From the 1016 OCSCC samples tested for HPV DNA, 940 (92.5\%) samples contained DNA of sufficient quality for a reliable HPV DNA test result as determined by successful detection of the human $\beta$-globin gene. From these 940 OCSCCs, 919 tumors were HPV DNAnegative and 21 (2.2\%) were HPV DNA-positive, of which 17 (81.0\%) for HPV16 (Figure 2). All HPV DNA-positive OCSCCs were subsequently tested for HPV type-specific E6 mRNA. All HPV DNA-positive tumors turned out to be HPV RNA-positive as well. To verify that the HPV-Risk Assay did not miss any HPV-positive OCSCC (ie, false negative HPV DNA test results), we additionally tested 50 randomly selected HPV DNA-negative OCSCC and tested these for HPV16 mRNA, as HPV16 is the HPV type most frequently detected. All HPV DNA-negative cases were confirmed to be HPV16 mRNA-negative.

\subsection{Demographic and clinical characteristics of HPV-negative and HPV-positive OCSCCs}

Demographic and clinical characteristics of the HPV-negative and HPVpositive OCSCCs are depicted in Table 1. Patients with HPV-positive OCSCCs were more frequently male $(95.2 \%$ vs $57.8 \%, P<.001)$ and younger than patients with HPV-negative carcinomas (59 vs 64 years, 


\section{Attributable fraction of high-risk HPV in oral cavity squamous cell carcinoma is $2.2 \%$}
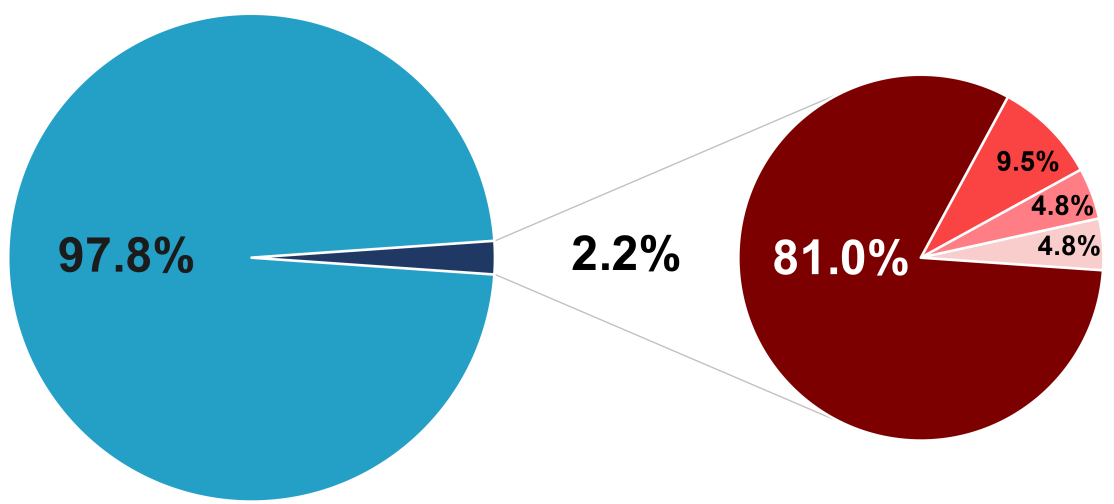

- High-risk HPV-negative ( $N=919)$

- HPV16-positive $(N=17)$

- HPV33-positive $(N=2)$

HPV45-positive $(N=1)$

HPV59-positive $(N=1)$

FIGURE 2 Prevalence of high-risk human papillomavirus (HPV) in oral cavity squamous cell carcinoma (OCSCC) [Color figure can be viewed at wileyonlinelibrary.com]

$P=.05)$. Although the majority of HPV-negative tumors were located in the mobile tongue, more than half of the HPV-positive tumors were found in the floor of the mouth. In total, $49.7 \%$ of HPV-negative tumors were treated with surgery alone and did not receive adjuvant treatment, whereas almost half of the HPV-positive tumors received postoperative radiotherapy, and $14.3 \%$ were treated with definitive chemoradiotherapy compared to $2.0 \%$ of the HPV-negative tumors.

\section{3 | Pathological characteristics of HPV-negative and HPV-positive OCSCCs}

All available pathological features of the HPV-negative and HPVpositive OCSCCs are summarized in Table 2. No significant differences were observed between HPV-negative and HPV-positive tumors, except for pT stage.

\subsection{Clinicopathologic characterization of HPV- positive OCSCCS}

In the light of the recently published article by Rooper et al, ${ }^{30}$ we performed an additional morphologic analysis on the 21 HPV-positive OCSCCs. Six OCSCCs were nonkeratinizing and 14 OCSCCs were keratinizing. We identified four tumors resembling the warty variant as described by Rooper (Supplementary Figure 1). None of the HPVpositive OCSCCs had adjacent (Bowenoid) CIS.

\section{5 | Prognostic impact of HPV in OCSCC}

From the 940 patients with a valid HPV test result, 881 (93.7\%) were treated with curative intent and were therefore included in the survival analysis. Median follow-up was 48 months. In Figure 3 , the Kaplan-Meier curves of the OS (Figure 3A) and DFS (Figure 3B) are shown for HPV-negative $(N=861)$ and HPV-positive $(N=20)$ OCSCCs. Five-year OS did not significantly differ between HPV- negative and HPV-positive OCSCCs (60.3\% vs $45.3 \%, P=.255)$, and the same was true for 5 -year DFS $(52.7 \%$ vs $45.3 \%, P=.685)$.

\section{6 | p16-status as surrogate marker for HPV in OCSCC}

p16-IHC was performed on 580 OCSCCs including all $21 \mathrm{HPV}$-positive cases and 559 randomly selected HPV-negative cases. In total, 534 (92.1\%) OCSCCs were p16-negative and 46 (7.6\%) were p16-positive. All p16-negative OCSCCs were HPV-negative and all HPV-positive OCSCCs were p16-positive. From the 46 p16-positive OCSCCs, 25 were HPV-negative, resulting in a sensitivity, specificity, positive and negative predictive value of $\mathrm{p} 16-\mathrm{IHC}$ as surrogate marker for a transforming HPV-infection of $100 \%, 95.5 \%, 45.7 \%$ and $100 \%$, respectively.

It was observed that the p16-staining pattern in OCSCC was more diverse than what is usually observed in OPSCC. OPSCCs are generally either completely p16-negative or convincingly p16-positive, with an obvious combination of nuclear and cytoplasmic immunoreactivity. In the current OCSCC cohort, however, some OCSCCs only showed nuclear immunostaining (Supplementary Figure 2A), while in other OCSCCs, the cytoplasm was exclusively immunopositive (Supplementary Figure 2B). Moreover, some tumors had weak immunostaining in the center of the tumor and strong immunostaining in the periphery (Supplementary Figure 2C). These varying patterns of immunostaining were not associated with the tumor differentiation grade or HPV DNA status (data not shown). Since these tumors did not meet the criterion for p16-positive immunostaining, they were considered p16-negative. All truly HPV-positive tumors displayed a convincing p16-immunostaining pattern with $>70 \%$ of the tumor cells.

\section{7 | p16-status as independent prognostic marker in OCSCC}

In Figure 4, the Kaplan-Meier curves for 5-year OS (Figure 4A) and DFS (Figure 4B) are shown for p16-negative and p16-positive OCSCCs. 
TABLE 1 Demographic and clinical characteristics of the cohort, as stratified by HPV-status

\begin{tabular}{|c|c|c|c|c|c|c|c|c|}
\hline & & \multicolumn{3}{|c|}{ HPV-negative OCSCCs } & \multicolumn{4}{|c|}{ HPV-positive OCSCCs } \\
\hline & & \multicolumn{3}{|l|}{$(N=919)$} & \multicolumn{4}{|l|}{$(\mathrm{N}=21)$} \\
\hline & & Mean (SD) & $\mathbf{N}$ & $\%$ & Mean (SD) & $\mathbf{N}$ & $\%$ & $P$ value \\
\hline Age at diagnosis & & $64(12)$ & & & $59(10)$ & & & $.050^{a}$ \\
\hline \multirow[t]{3}{*}{ Sex } & & & & & & & & $<.001^{\mathrm{b}}$ \\
\hline & Male & & 531 & 57.8 & & 20 & 95.2 & \\
\hline & Female & & 388 & 42.2 & & 1 & 4.8 & \\
\hline \multirow[t]{5}{*}{ ACE-27 } & & & & & & & & $.397^{\mathrm{b}}$ \\
\hline & None & & 287 & 31.2 & & 10 & 47.6 & \\
\hline & Mild & & 303 & 33.0 & & 4 & 19.0 & \\
\hline & Moderate & & 226 & 24.6 & & 5 & 23.8 & \\
\hline & Severe & & 103 & 11.2 & & 2 & 9.5 & \\
\hline Pack years & & $28(26)$ & & & $34(20)$ & & & $.146^{\mathrm{a}}$ \\
\hline Unit years & & $131(158)$ & & & $167(159)$ & & & $.260^{\mathrm{a}}$ \\
\hline \multirow[t]{7}{*}{ Tumor location } & & & & & & & & $.018^{c}$ \\
\hline & Mobile tongue & & 415 & 45.2 & & 6 & 28.6 & \\
\hline & Floor of mouth & & 256 & 27.9 & & 12 & 57.1 & \\
\hline & Vestibulum of mouth & & 122 & 13.3 & & 0 & 0.0 & \\
\hline & Hard palate & & 12 & 1.3 & & 1 & 4.8 & \\
\hline & Cheek mucosa & & 70 & 7.6 & & 2 & 9.8 & \\
\hline & Retromolar trigone & & 44 & 4.8 & & 0 & 0.0 & \\
\hline \multirow[t]{7}{*}{ cT stage (TNM-8) } & & & & & & & & $.096^{c}$ \\
\hline & Tis & & 9 & 1.0 & & 1 & 4.8 & \\
\hline & T1 & & 348 & 37.9 & & 3 & 14.3 & \\
\hline & $\mathrm{T} 2$ & & 290 & 31.6 & & 9 & 42.9 & \\
\hline & T3 & & 66 & 7.2 & & 2 & 9.5 & \\
\hline & $\mathrm{T} 4 \mathrm{a}$ & & 188 & 20.5 & & 6 & 28.6 & \\
\hline & $\mathrm{T} 4 \mathrm{~b}$ & & 18 & 2.0 & & 0 & 0.0 & \\
\hline \multirow[t]{8}{*}{ cN stage (TNM-8) } & & & & & & & & $.553^{\mathrm{c}}$ \\
\hline & NO & & 723 & 78.7 & & 16 & 76.2 & \\
\hline & N1 & & 91 & 9.9 & & 4 & 19.0 & \\
\hline & $\mathrm{N} 2 \mathrm{a}$ & & 4 & 0.4 & & 0 & 0.0 & \\
\hline & $\mathrm{N} 2 \mathrm{~b}$ & & 48 & 5.2 & & 0 & 0.0 & \\
\hline & $\mathrm{N} 2 \mathrm{c}$ & & 36 & 3.9 & & 1 & 4.8 & \\
\hline & N3a & & 0 & 0.0 & & 0 & 0.0 & \\
\hline & N3b & & 17 & 1.8 & & 0 & 0.0 & \\
\hline \multirow[t]{8}{*}{ Treatment received } & & & & & & & & $.014^{\mathrm{c}}$ \\
\hline & None & & 37 & 5.0 & & 1 & 4.8 & \\
\hline & Surgery & & 457 & 49.7 & & 5 & 23.8 & \\
\hline & Surgery + RT & & 309 & 33.6 & & 10 & 47.6 & \\
\hline & Surgery + CRT & & 40 & 4.4 & & 0 & 0.0 & \\
\hline & Radiotherapy & & 46 & 5.0 & & 2 & 9.5 & \\
\hline & Chemoradiotherapy & & 18 & 2.0 & & 3 & 14.3 & \\
\hline & Other & & 12 & 1.3 & & 0 & 0.0 & \\
\hline
\end{tabular}

Abbreviations: CRT, chemoradiotherapy; HPV, human papillomavirus; OCSCC, oral cavity squamous cell carcinoma; RT, radiotherapy. aMann-Whitney $U$ test.

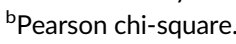

'Fisher's exact test. 
TABLE 2 Pathological characteristics of the cohort, as stratified by HPV-status

\begin{tabular}{|c|c|c|c|c|c|c|c|c|}
\hline & & \multicolumn{3}{|c|}{ HPV-negative OCSCCs } & \multicolumn{4}{|c|}{ HPV-positive OCSCCs } \\
\hline & & Mean (SD) & $N$ & $\%$ & Mean (SD) & $\mathbf{N}$ & $\%$ & $P$ value \\
\hline Tumor diameter $(\mathrm{cm})$ & & $2.21(1.46)$ & & & $2.69(1.97)$ & & & $.518^{\mathrm{a}}$ \\
\hline Tumor depth of invasion $(\mathrm{cm})$ & & $0.82(0.78)$ & & & $1.01(1.23)$ & & & $.576^{\mathrm{a}}$ \\
\hline \multirow{2}{*}{ Tumor pattern of invasion } & Noncohesive & & 450 & 49.0 & & 8 & 38.1 & \\
\hline & Unknown & & 154 & 16.8 & & 5 & 23.8 & \\
\hline \multirow[t]{2}{*}{ Lymphovascular invasion } & & & & & & & & $.327^{c}$ \\
\hline & No & & 658 & 71.6 & & 13 & 61.9 & \\
\hline \multirow{3}{*}{ Perineural invasion } & No & & 567 & 61.7 & & 12 & 57.1 & \\
\hline & Yes & & 209 & 22.7 & & 3 & 14.3 & \\
\hline & Unknown & & 143 & 15.6 & & 6 & 28.6 & \\
\hline \multirow[t]{5}{*}{ Differentiation grade } & & & & & & & & $.238^{\mathrm{c}}$ \\
\hline & Well differentiated & & 129 & 14.0 & & 1 & 4.8 & \\
\hline & Moderately differentiated & & 481 & 52.3 & & 16 & 76.2 & \\
\hline & Poorly differentiated & & 196 & 21.3 & & 2 & 9.5 & \\
\hline & Unknown & & 113 & 12.3 & & 2 & 9.5 & \\
\hline \multirow[t]{3}{*}{ pT stage (TNM-8) } & & & & & & & & $.048^{c}$ \\
\hline & n.a. (no surgery) & & 110 & 12.0 & & 6 & 28.6 & \\
\hline & Tx (not assessable) & & 4 & 0.4 & & 0 & 0.0 & \\
\hline \multirow[t]{10}{*}{ pN stage (TNM-8) } & & & & & & & & $.794^{c}$ \\
\hline & NO & & 378 & 41.1 & & 9 & 42.9 & \\
\hline & N1 & & 89 & 9.7 & & 1 & 4.8 & \\
\hline & $\mathrm{N} 2 \mathrm{a}$ & & 29 & 3.2 & & 0 & 0.0 & \\
\hline & $\mathrm{N} 2 \mathrm{~b}$ & & 43 & 4.7 & & 1 & 4.8 & \\
\hline & $\mathrm{N} 2 \mathrm{c}$ & & 14 & 1.5 & & 1 & 4.8 & \\
\hline & N3a & & 0 & 0.0 & & 0 & 0.0 & \\
\hline & N3b & & 83 & 9.0 & & 2 & 9.5 & \\
\hline & n.a. (no neck dissection) & & 282 & 30.7 & & 7 & 33.3 & \\
\hline & Nx (not assessable) & & 1 & 0.1 & & 0 & 0.0 & \\
\hline \multirow[t]{5}{*}{ Extranodal extension } & & & & & & & & $1.000^{c}$ \\
\hline & No & & 524 & 57.0 & & 12 & 57.1 & \\
\hline & Yes & & 112 & 12.2 & & 2 & 9.5 & \\
\hline & n.a. (no neck dissection) & & 282 & 30.7 & & 7 & 33.3 & \\
\hline & Unknown & & 1 & 0.1 & & 0 & 0.0 & \\
\hline Surgical margins & & & & & & & & $.189^{c}$ \\
\hline
\end{tabular}


TABLE 2 (Continued)

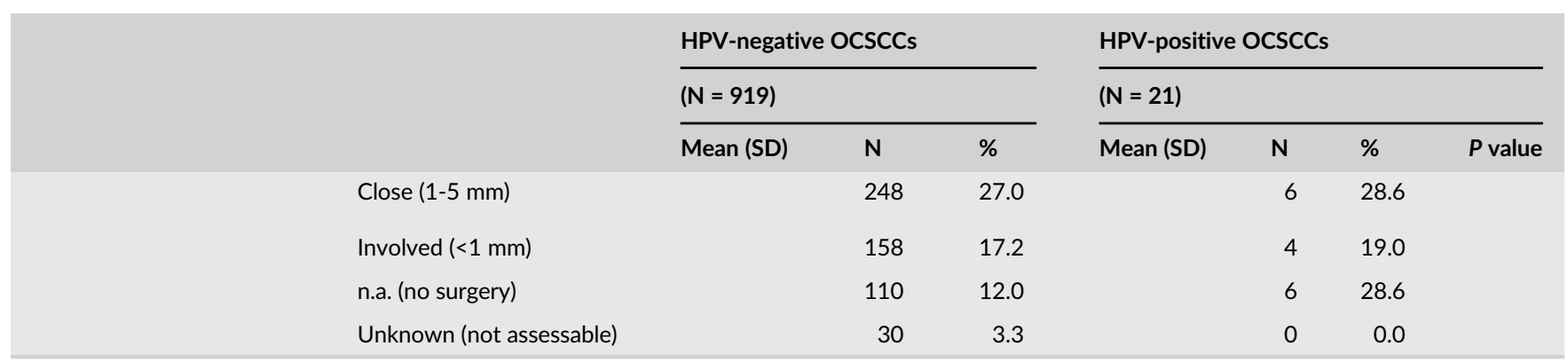

Abbreviations: HPV, human papillomavirus; OCSCC, oral cavity squamous cell carcinoma.

aMann-Whitney $U$ test.

${ }^{b}$ Pearson chi-square.

'Fisher's exact test.

Five-year OS did not significantly differ between p16-negative and p16-positive OCSCCs (58.2\% vs $51.9 \%, P=.482)$, and the same was true for 5 -year DFS (53.2\% vs $41.9 \%$, respectively; $P=.262$ ).

\section{4 | DISCUSSION}

To our knowledge, this is the first large study in which the attributable fraction of high-risk HPV in a consecutive cohort of almost a thousand OCSCCs from a European country is reported. We took several precautions to obtain the most accurate data. First, tumors crossing the anatomical borders of the oropharynx and oral cavity were excluded as this could impact both attributable fraction and prognosis. Next, we analyzed a large consecutive cohort of 940 cases to obtain convincing figures. HPV-attributions were comparable between Amsterdam UMC (2.0\%) and Erasmus MC Rotterdam (2.4\%) Third, HPV-testing was performed using the validated HPV-rTcore workflow, ${ }^{28}$ with H\&E-guided core punch biopsies taken from the identified tumor area with a disposable device. This enabled us to omit sectioning of FFPE-blocks on the microtome, which is laborious and carries the risk of sample cross-contamination. Although little literature has been published on molecular contamination of HPV during sectioning, this phenomenon is described for Bartonella and simian herpesviruses. ${ }^{33,34}$

HPV DNA-status was established with an E7-based assay, which overcomes potential false-negative results caused by viral integration with accompanying loss of HPV-regions targeted by most other HPV-assays, such as those targeting L1. The assay is validated to detect clinically relevant HPV-infection in the cervix, ${ }^{31,35,36}$ and its sensitivity has been limited on purpose. All positive test results were verified by appropriate HPV type-specific mRNA-tests, strongly indicating that HPV DNA-assays can be used with optimally designed procedures, despite the fact that we did not wipe the surface of the blocks before punching. With ultrasensitive DNA-tests, such as the SPF-10, this picture might change. Now we observed a 100\% concordance between DNA- and RNA-based testing, implying that additional mRNA-testing of HPV DNA-positive OCSCCs can be omitted when applying the DNA-assay used in our study.

The HPV-attribution in our OCSCC cohort was a mere $2.2 \%$, which is lower than HPV-attributable fractions reported in several other studies. When exploring the existing literature, the prevalence of transcriptionally active HPV in OCSCC varies between $2.0 \%$ and $61 \%{ }^{1,2,12,14-22}$ The HPV-attribution found in the current study likely relates to the strict criteria that were applied, but could also in part be explained by geographical variation. Castellsagué and colleagues reported data in OCSCC of $6.6 \%$ in Central-South America, $3.7 \%$ in Europe and $0 \%$ in Africa and Asia. ${ }^{1}$ Hence, geographical variation does occur. Moreover, HPV-attribution could change in time as has been noted in OPSCC as well. ${ }^{27}$ However, we did neither find variation between the two Dutch centers, nor any trend of increasing prevalence over time (Supplementary Figure 3).

Only few studies describe in detail the clinical characteristics of OCSCCs harboring transcriptionally active HPV. In their meta-analysis, Ndiaye et al found the highest HPV-prevalence within the oral cavity to be in carcinomas arising from the "palate not otherwise specified" (C05.0-9, HPV-prevalence 42.6\%), the "gum" (C03.0-9, HPV-prevalence 39.9\%) and the "tongue not otherwise specified (C01, C02.0-9, HPV-prevalence $28.0 \%)^{2}$ Unfortunately, these HPV-attributions are based on HPV DNA PCR, and could therefore just as well reflect productive HPV-infections. On the other hand, Bussu et al tested 33 OCSCCs for the presence of HPV, and found one tumor to be HPV18 mRNA-positive. ${ }^{14}$ This tumor was located in the floor of mouth. Lingen et al tested 409 OCSCC for the presence of transcriptionally active HPV, and found 24 (5.9\%) OCSCCs to be HPV mRNA-positive. Nine (37.5\%) of these tumors were located in the floor of mouth, six (25.0\%) in the tongue, and four (16.7\%) in the alveolar process. ${ }^{16}$ Hernandez et al studied 122 OCSCC, of which 38 (31.1\%) were HPV DNA-positive. Seventeen (45\%) of HPV DNA-positive OCSCCs were located in the oral tongue and $10(26 \%)$ in the floor of mouth.

In several studies, the effect of p16-status and/or HPV-status in OCSCCs was investigated. In some studies it was found that OS did not vary by HPV DNA-positivity, p16-positivity or joint positivity for both markers. ${ }^{17,18,20}$ In contrast to the current study, two studies by Chung et al and Bryant et al found p16-status to play a comparable prognostic role in both oropharyngeal and nonoropharyngeal cancer, including OCSCC. ${ }^{12,29}$

Finally, we investigated the performance of $\mathrm{p} 16-\mathrm{IHC}$ as surrogate marker for a transforming HPV-infection, and assessed the 
(A)

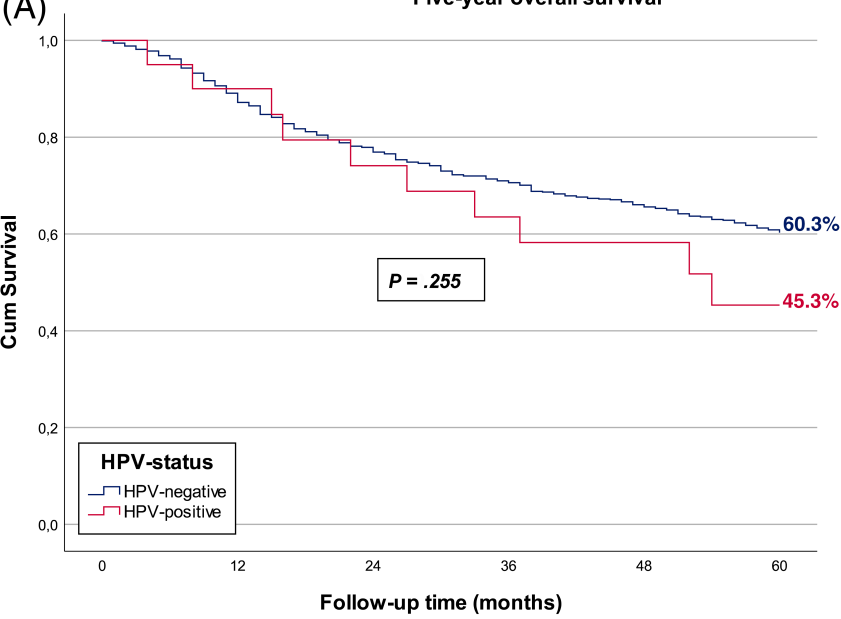

(B)

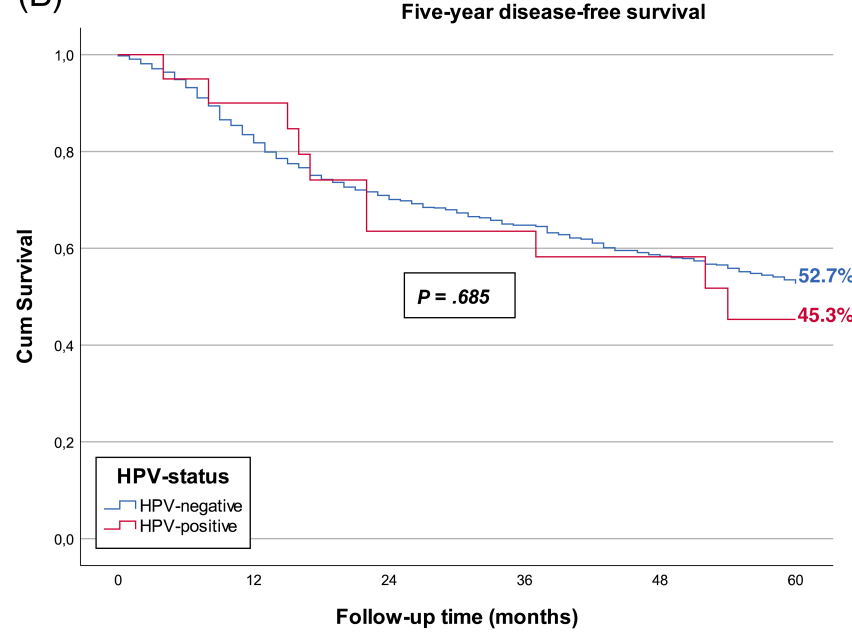

FIGURE 3 Kaplan-Meier curves of human papillomavirus (HPV)negative and HPV-positive oral cavity squamous cell carcinomas (OCSCCs). A, Overall survival (OS) of HPV-negative (blue line, 5-year OS 60.3\%) and HPV-positive (red line, 5-year OS 45.3\%) OCSCC patients. Log-rank analysis showed no difference in OS between HPV-negative and HPV-positive OCSCCs $(P=.255)$. B, Disease-free survival (DFS) of HPV-negative (blue line, 5-year DFS 52.7\%) and HPV-positive (red line, 5-year DFS 45.3\%) OCSCC patients. Log-rank analysis showed no difference in DFS between HPV-negative and HPV-positive OCSCCs $(P=.685)$ [Color figure can be viewed at wileyonlinelibrary.com]

independent prognostic impact of p16-positivity in OCSCC. P16-IHC was carried out on 580 OCSCCs, of which 534 (92.1\%) OCSCCs were p16-negative and 46 OCSCCs p16-positive. From the 46 p16-positive OCSCCs, 21 (45.7\%) were HPV-positive and 25 (54.3\%) were HPVnegative. To investigate whether the 25 p16-positive/HPV-negative OCSCCs had a false-positive p16-status or a false-negative HPV-status, all cases were additionally subjected to HPV16 mRNA-testing. All 25 OCSCCs remained HPV-negative when tested with for HPV16 mRNA-testing. The sensitivity, specificity, positive and negative predictive value of p16-status as surrogate marker for a transforming HPV-infection were 100\%, 95.5\%, 45.7\% and 100\%, respectively,

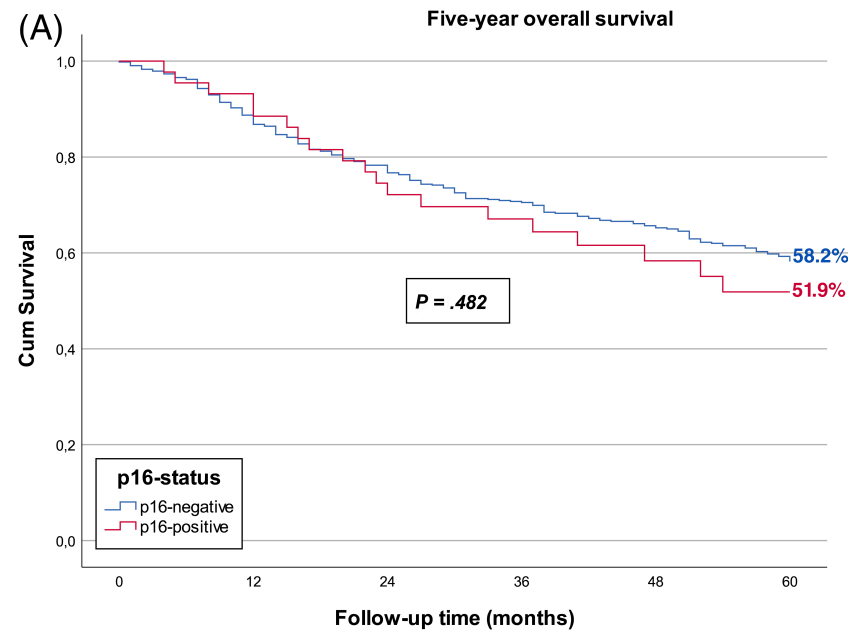

(B)

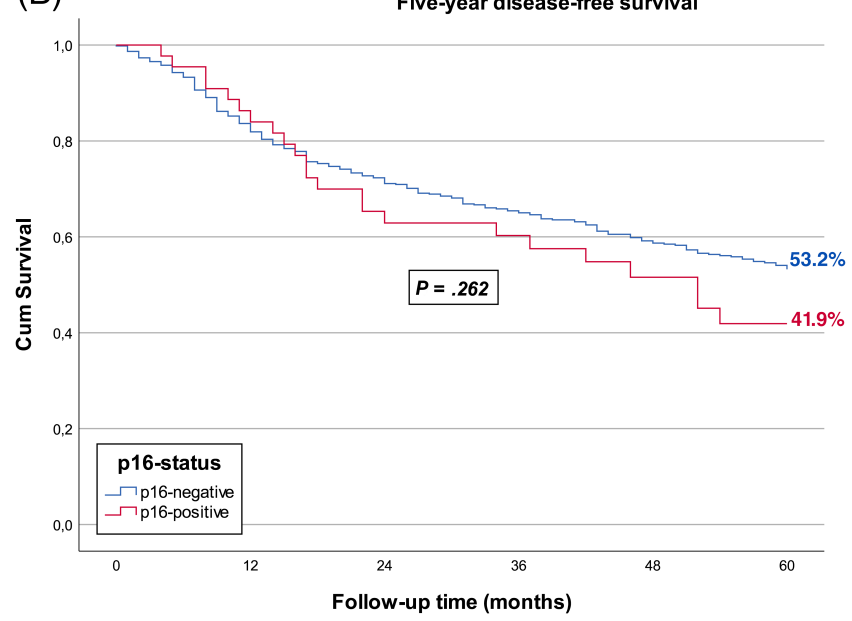

FIGURE 4 Kaplan-Meier curves of p16-negative and p16-positive oral cavity squamous cell carcinomas (OCSCCs). A, Overall survival (OS) of p16-negative (blue line, 5-year OS 58.2\%) and p16-positive (red line, 5-year OS 51.9\%) OCSCC patients. Logrank analysis showed no difference in OS between $\mathrm{p} 16$-negative and p16-positive OCSCCs $(P=.482)$. B, Disease-free survival (DFS) of p16-negative (blue line, 5-year DFS 53.2\%) and p16-positive (red line, 5-year DFS 41.9\%) OCSCC patients. Log-rank analysis showed no difference in DFS between p16-negative and p16-positive OCSCCs $(P=.262)$ [Color figure can be viewed at wileyonlinelibrary.com]

which is comparable to the values reported by Lingen et al. ${ }^{16}$ Still, it should be acknowledged that the positive and negative predictive values of a test always relate to the disease prevalence rate in a population and may thus be different in other populations. Because of the low positive predictive value, $\mathrm{p} 16-\mathrm{IHC}$ alone is not sufficient to establish HPV-status in OCSCC. However, given the sensitivity and negative predictive value of $100 \%$, it can be used as a first screening to determine which OCSCCs qualify for subsequent HPV-testing. Although some studies found a survival benefit of p16-positivity in OCSCC, ${ }^{12,29}$ we did not find any impact of $\mathrm{p} 16$-status on prognosis. Our findings are in line with other studies investigating the prognostic potential of p16-status in ocscC. $^{18}$ of note, it should be 
acknowledged that the criteria for p16-positivity in OCSCC applied in our study-and in the literature in general-are actually set and validated for OPSCCs. The oral cavity is an independent site of the head and neck area, and the composition of the oral squamous epithelium is different from the oropharyngeal epithelium (eg, less lymphoid tissue). Moreover, in the current study it was noticed that OCSCCs display a more diverse p16-staining pattern when compared to OPSCCs. The cutoff value for p16-positivity used in OPSCC seems to be well applicable to OCSCC, albeit a subsequent HPV DNA-test is absolutely required given the suboptimal positive predictive value.

When comparing the HPV-positive OCSCCs to the HPV-negative OCSCCs, we found some remarkable differences in clinical characteristics (Table 1). For example, $49.7 \%$ of HPV-negative tumors were treated with surgery alone and did not receive adjuvant treatment, whereas almost half of the HPV-positive tumors received postoperative radiotherapy, and $14.3 \%$ were treated with definitive chemoradiotherapy compared to $2.0 \%$ of the HPV negative tumors. As depicted in Table 1, HPV-positive OCSCCs tended to have a higher T-stage than HPV-negative tumors. This may in part be explained by the fact that the majority of HPV-positive OCSCCs were located in the floor of the mouth, often with invasion of the mandible. These tumors require postoperative radiotherapy. What is more, $15 \%$ of HPV-positive tumors were too large to be treated with surgery, that is, functionally inoperable tumors. These tumors were therefore treated with definitive chemoradiotherapy.

To conclude, HPV-attribution in OCSCC is very low and neither HPV-status nor p16-status affects survival of OCSCC patients. Although when followed by a PCR test p16-IHC may fairly well serve as a first step in assessing HPV-status, determining HPV-status in OCSCC seems trivial in clinical practice and should be omitted.

\section{ACKNOWLEDGMENTS}

We thank Martijn Bogaarts, Dénira Agard, Marjolein Bekker-Lettink, Mark Broeckaert, Douwe Buma, Marinda de Groot-van Moorsel and Marije Graafmans-Doeleman for their inexhaustible efforts to test more than a thousand OCSCCs for HPV. This study was funded by the European Union (H2020 Grant Agreement 689715 BD2Decide).

\section{CONFLICT OF INTEREST}

Daniëlle A. M. Heideman has been on the speakers' bureau of QIAGEN and serves occasionally on the scientific advisory boards of Pfizer and Bristol-Myers Squibb; Daniëlle A.M. Heideman is minority shareholder of Self-screen B.V., a spin-off company of VUmc; Self-screen B.V. holds patents related to the work (ie, high-risk HPV test and methylation markers). C. René Leemans has been on the advisory boards of Merck \& Co. Inc., Rakuten Medical, and Nanobiotix, and reports research grants from KWF Kankerbestrijding/Dutch Cancer Society, VUmc Cancer Center Amsterdam Foundation, GenMab, BMS and the Hanarth Foundation, outside the submitted work. Ruud H. Brakenhoff reports grants from GenMab, InteRNA technologies, and Agilent and nonfinancial support from AbbVie, outside the submitted work. All the other authors declared no potential conflicts of interest.

\section{DATA AVAILABILITY STATEMENT}

The data that support the findings of this study are available from the corresponding author (rh.brakenhoff@amsterdamumc.nl) upon reasonable request.

\section{ETHICS STATEMENT}

The study adhered to the national guideline "Human Tissue and Medical Research: Code of Conduct for Responsible Use" (https://www. federa.org/codes-conduct), and the general privacy protection regulation of the European Commission.

\section{ORCID}

Irene H. Nauta (D) https://orcid.org/0000-0003-0318-715X

Ruud H. Brakenhoff (D) https://orcid.org/0000-0003-3251-5378

\section{REFERENCES}

1. Castellsague X, Alemany L, Quer M, et al. HPV involvement in head and neck cancers: comprehensive assessment of biomarkers in 3680 patients. J Natl Cancer Inst. 2016;108(6):djv403.

2. Ndiaye C, Mena M, Alemany L, et al. HPV DNA, E6/E7 mRNA, and p16INK4a detection in head and neck cancers: a systematic review and meta-analysis. Lancet Oncol. 2014;15(12):1319-1331.

3. Anantharaman D, Abedi-Ardekani B, Beachler DC, et al. Geographic heterogeneity in the prevalence of human papillomavirus in head and neck cancer. Int J Cancer. 2017;140(9):1968-1975.

4. Haeggblom L, Ramqvist T, Tommasino M, Dalianis T, Nasman A. Time to change perspectives on HPV in oropharyngeal cancer. A systematic review of HPV prevalence per oropharyngeal sub-site the last 3 years. Papillomavirus Res. 2017;4:1-11.

5. Woods RSR, Keegan $\mathrm{H}$, White $\mathrm{C}$, et al. Cytokeratin 7 in oropharyngeal squamous cell carcinoma: a Junctional biomarker for human papillomavirus-related tumors. Cancer Epidemiol Biomarkers Prev. 2017;26(5):702-710.

6. Chaturvedi AK, Engels EA, Anderson WF, Gillison ML. Incidence trends for human papillomavirus-related and -unrelated oral squamous cell carcinomas in the United States. J Clin Oncol. 2008;26(4): 612-619.

7. Gillison ML, D'Souza G, Westra W, et al. Distinct risk factor profiles for human papillomavirus type 16-positive and human papillomavirus type 16-negative head and neck cancers. J Natl Cancer Inst. 2008;100 (6):407-420

8. Rietbergen $\mathrm{MM}$, Brakenhoff $\mathrm{RH}$, Bloemena $\mathrm{E}$, et al. Human papillomavirus detection and comorbidity: critical issues in selection of patients with oropharyngeal cancer for treatment De-escalation trials. Ann Oncol. 2013;24(11):2740-2745.

9. Ang $\mathrm{KK}$, Harris J, Wheeler R, et al. Human papillomavirus and survival of patients with oropharyngeal cancer. N Engl J Med. 2010;363(1): 24-35.

10. O'Sullivan B, Huang SH, Su J, et al. Development and validation of a staging system for HPV-related oropharyngeal cancer by the international collaboration on oropharyngeal cancer network for staging (ICON-S): a multicentre cohort study. Lancet Oncol. 2016;17(4): 440-451.

11. Rodrigo JP, Hermsen MA, Fresno MF, et al. Prevalence of human papillomavirus in laryngeal and hypopharyngeal squamous cell carcinomas in northern Spain. Cancer Epidemiol. 2015;39(1):37-41.

12. Chung $\mathrm{CH}$, Zhang $\mathrm{Q}$, Kong $\mathrm{CS}$, et al. $\mathrm{p} 16$ protein expression and human papillomavirus status as prognostic biomarkers of nonoropharyngeal head and neck squamous cell carcinoma. J Clin Oncol. 2014;32(35):3930-3938. 
13. Wendt M, Romanitan M, Nasman A, et al. Presence of human papillomaviruses and p16 expression in hypopharyngeal cancer. Head Neck. 2014;36(1):107-112.

14. Bussu F, Sali M, Gallus R, et al. HPV infection in squamous cell carcinomas arising from different mucosal sites of the head and neck region. Is p16 immunohistochemistry a reliable surrogate marker? $\mathrm{Br} J$ Cancer. 2013;108(5):1157-1162.

15. Dediol E, Sabol I, Virag M, Grce M, Muller D, Manojlovic S. HPV prevalence and p16INKa overexpression in non-smoking non-drinking oral cavity cancer patients. Oral Dis. 2016;22(6):517-522

16. Lingen $M W$, Xiao $W$, Schmitt $A$, et al. Low etiologic fraction for highrisk human papillomavirus in oral cavity squamous cell carcinomas. Oral Oncol. 2013;49(1):1-8.

17. Fakhry C, Westra WH, Wang SJ, et al. The prognostic role of sex, race, and human papillomavirus in oropharyngeal and nonoropharyngeal head and neck squamous cell cancer. Cancer. 2017;123(9):1566-1575.

18. Hernandez BY, Lynch CF, Chan OTM, et al. Human papillomavirus DNA detection, p16(INK4a), and oral cavity cancer in a U.S. population. Oral Oncol. 2019;91:92-96.

19. Herrero R, Castellsague $X$, Pawlita $M$, et al. Human papillomavirus and oral cancer: the International Agency for Research on Cancer multicenter study. J Natl Cancer Inst. 2003;95(23):1772-1783.

20. Lai K, Killingsworth M, Matthews S, et al. Differences in survival outcome between oropharyngeal and oral cavity squamous cell carcinoma in relation to HPV status. J Oral Pathol Med. 2017;46(8):574-582.

21. de Martel C, Plummer M, Vignat J, Franceschi S. Worldwide burden of cancer attributable to HPV by site, country and HPV type. Int J Cancer. 2017;141(4):664-670.

22. Zafereo ME, Xu L, Dahlstrom KR, et al. Squamous cell carcinoma of the oral cavity often overexpresses $\mathrm{p} 16$ but is rarely driven by human papillomavirus. Oral Oncol. 2016;56:47-53.

23. Chi AC, Day TA, Neville BW. Oral cavity and oropharyngeal squamous cell carcinoma-an update. CA Cancer J Clin. 2015;65(5):401-421.

24. Boscolo-Rizzo P, Pawlita M, Holzinger D. From HPV-positive towards HPV-driven oropharyngeal squamous cell carcinomas. Cancer Treat Rev. 2016;42:24-29.

25. Leemans CR, Snijders PJF, Brakenhoff RH. The molecular landscape of head and neck cancer. Nat Rev Cancer. 2018;18(5):269-282.

26. Smeets SJ, Hesselink AT, Speel EJ, et al. A novel algorithm for reliable detection of human papillomavirus in paraffin embedded head and neck cancer specimen. Int J Cancer. 2007;121(11):2465-2472.

27. Rietbergen $M M$, Leemans CR, Bloemena $E$, et al. Increasing prevalence rates of HPV attributable oropharyngeal squamous cell carcinomas in the Netherlands as assessed by a validated test algorithm. Int J Cancer. 2013;132(7):1565-1571.
28. Mes SW, Heideman DAM, Bloemena E, et al. Development and validation of a novel and rapid molecular detection method for high-risk human papillomavirus in formalin-fixed paraffin-embedded tumor tissue. J Mol Diagn. 2020;22(2):262-271.

29. Bryant AK, Sojourner EJ, Vitzthum LK, et al. Prognostic role of p16 in nonoropharyngeal head and neck cancer. J Natl Cancer Inst. 2018;110 (12):1393-1399.

30. Rooper LM, Windon MJ, Hernandez T, et al. HPV-positive squamous cell carcinoma of the larynx, oral cavity, and hypopharynx: clinicopathologic characterization with recognition of a novel warty variant. Am J Surg Pathol. 2020;44(5):691-702.

31. Hesselink AT, Berkhof J, van der Salm ML, et al. Clinical validation of the HPV-risk assay, a novel real-time PCR assay for detection of high-risk human papillomavirus DNA by targeting the E7 region. J Clin Microbiol. 2014;52(3):890-896.

32. Schmitt M, Bravo IG, Snijders PJ, Gissmann L, Pawlita M, Waterboer T. Bead-based multiplex genotyping of human papillomaviruses. J Clin Microbiol. 2006;44(2):504-512.

33. Varanat M, Maggi RG, Linder KE, Horton S, Breitschwerdt EB. Crosscontamination in the molecular detection of Bartonella from paraffinembedded tissues. Vet Pathol. 2009;46(5):940-944.

34. Ritchey JW, Black DH, Rogers KM, Eberle R. In vivo experimentation with simian herpesviruses: assessment of biosafety and molecular contamination. J Am Assoc Lab Anim Sci. 2006;45(2):7-12.

35. Polman NJ, Ostrbenk A, Xu L, et al. Evaluation of the clinical performance of the HPV-risk assay using the VALGENT-3 panel. J Clin Microbiol. 2017;55(12):3544-3551.

36. Heideman DAM, Xu L, Hesselink AT, et al. Clinical performance of the HPV-risk assay on cervical samples in SurePath medium using the VALGENT-4 panel. J Clin Virol. 2019;121:104201.

\section{SUPPORTING INFORMATION}

Additional supporting information may be found online in the Supporting Information section at the end of this article.

How to cite this article: Nauta IH, Heideman DAM, Brink A, et al. The unveiled reality of human papillomavirus as risk factor for oral cavity squamous cell carcinoma. Int. J. Cancer. 2021;149:420-430. https://doi.org/10.1002/ijc.33514 